\title{
A Review of the South African National Oral Health Policy
}

SADJ October 2021, Vol. 76 No. 9 p551 - p557

Dr NA Mukhari-Baloyi ${ }^{1}$, Prof A Bhayat ${ }^{2}$, Prof TK Madiba ${ }^{3}$, Dr NR Nkambule ${ }^{4}$

\section{ABSTRACT}

\section{Introduction}

Advocacy for oral health promotion, prevention and treatment of oral diseases is founded on a comprehensive oral health policy that is integrated into the South African National Health Policy. The policy is intended to be a roadmap in the pursuit of achieving optimum oral health for the South African population.

\section{Aim}

To review the National Oral Health Policy of SA (NOHPSA) in terms of context, strengths, weaknesses, implementation and monitoring regarding the oral health status of the South African population.

\section{Results}

Promotive and preventive services lack detail on activities to achieve goals. The objectives, although very well defined, cannot be measured and there is no way of knowing whether they have been achieved. It was evident that the policy needs to be reviewed and updated in accordance to recent survey data, population growth and the profile (number and types) of health service providers. The prospect of updating the policy relies on the availability of current epidemiological surveys, which are not available, the most recent survey was conducted in 2002.

\section{Author affiliations:}

1. Dr Ntsakisi A Mukhari-Baloyi: BDS, Advanced Diploma (Community Dentistry). Registrar in the Department of Community Dentistry, School of Dentistry, University of Pretoria, Pretoria, South Africa. ORCID ID: 0000-0002-4374-0186

2. Prof Ahmed Bhayat: BDS, MSc, MPH, MDent (Community Dentistry). Head of Community Dentistry, Department of Community Dentistry, School of Dentistry, University of Pretoria, Pretoria, South Africa. ORCID ID:000-0002-8103-1233

3. Prof Thomas K Madiba: B Dent Ther, BDS, DHSM, MChD (Community Dentistry), Department of Community Dentistry, Schoo of Dentistry, University of Pretoria, Pretoria, South Africa. ORCID ID:0000-0002-0171-0595

4. Dr Ntombizodwa R Nkambule: $B$ Dent Ther, BDS, MPH, DHSM, $M C h D$ (Community Dentistry), Department of Community Dentistry, School of Dentistry, University of Pretoria, Pretoria, South Africa. ORCID ID: 0000-0003-2524-6413

Corresponding author: Dr N A Mukhari- Baloyi

Email: ntsakisi@gmail.com

Tel: 0123192531 / Cell: 0815489893

The role played and the respective contribution:

1. Dr Ntsakisi A. Mukhari-Baloyi: $40 \%$

2. Prof Ahmed Bhayat: $20 \%$

3. Prof Thomas K. Madiba: $20 \%$

4. Dr Ntombizodwa R. Nkambule: $20 \%$

\section{Conclusion}

The policy lacks a monitoring and evaluation plan. This is critical not only for the assessment of actualisation of aims and objectives, but for the sustainability of intended interventions.

\section{Keywords}

Oral health policy, national health policy

\section{INTRODUCTION}

The review of policies in health is important to determine their relevance and applicability over time and to determine whether the aims and goals are still being met. A review thus informs the recommendation for changes to improve the effectiveness and give clarity to indicators for desired outcomes. A thorough review requires an analysis of the implementation, monitoring and evaluation phases of the policy making process; this ensures that the final phase which is the maintenance, succession or termination of the policy can be decided on.

Oral health policies, like other health policies, are essential in any country as they provide direction as to where the current health status is, where they want to move it toward and the resources that will be required to get to their desired destination. Studies have shown that countries without any clear oral health policy, like Russia, tend to have a higher prevalence of oral disease in comparison to other countries with similar socio economic standings. ${ }^{1}$ This could be attributed to the fact that if there is a need to improve the health of any nation, there must be clear goals, a clear direction and a destination with time lines to ensure that all stakeholders, NGOs, and funding agencies can monitor the progress of the countries health at any given time. ${ }^{2}$

Oral health policies of SA, China and Brazil, countries with a similar economic rating, as they fall under the BRICS umbrella (Brazil, Russia, India, China and South Africa); are very similar in context but they vary significantly in their implementation, monitoring and evaluation. ${ }^{3-7}$ These countries' policies are all based on their respective National Health policies. ${ }^{8-12}$

The National Oral Health Policy of South Africa (NOHPSA) was formulated based on the National Health Policy of 1990. ${ }^{8}$ The policy is founded on two broad pillars; the goals that need to be achieved for oral health in SA and the targets that need to be met in terms of treating and preventing oral health diseases, as shown below (Figure 1). 


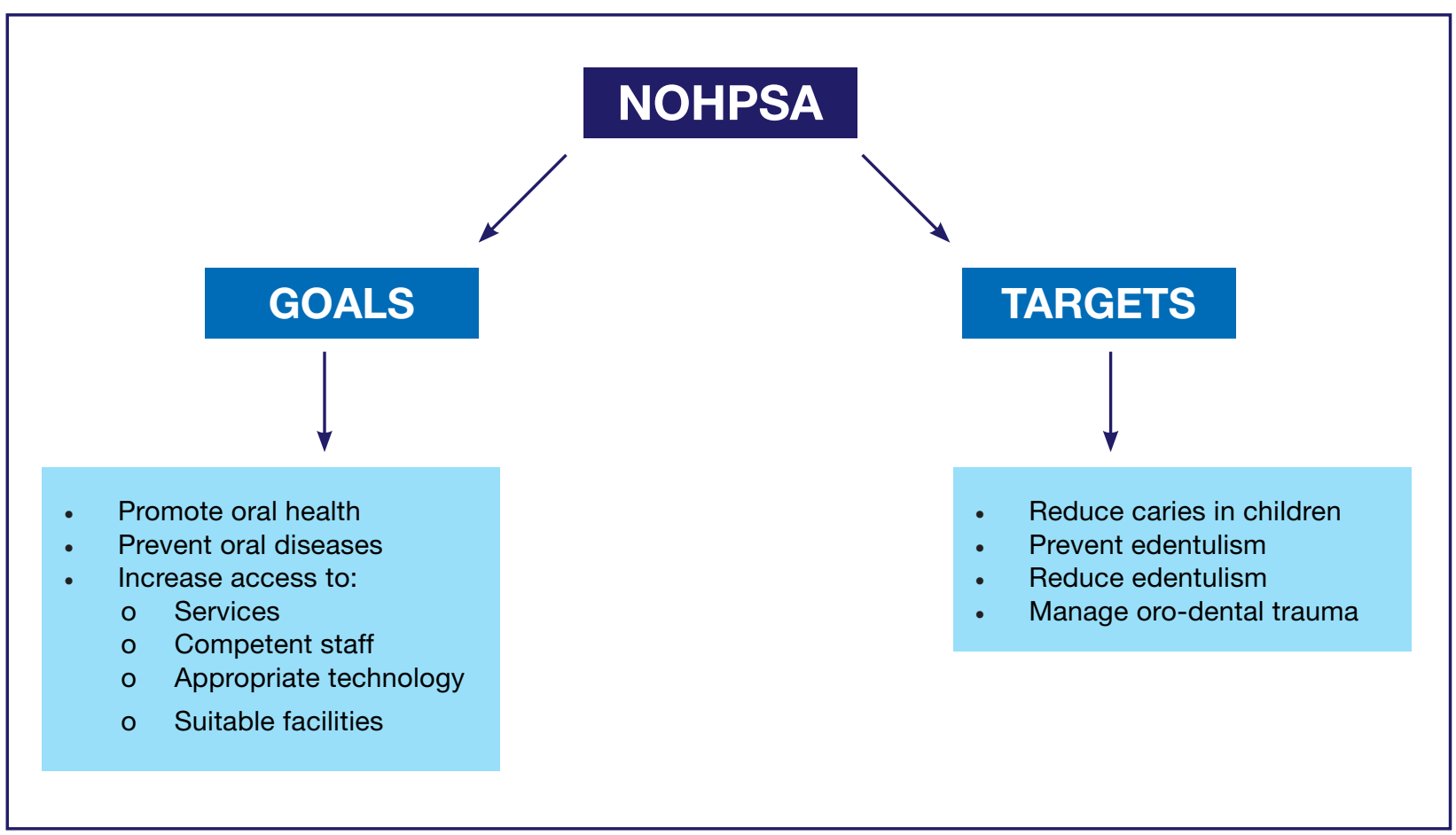

Figure 1. Diagrammatic representation of the goals and targets of the NOHPSA

Goals

The goals of the NOHPSA are to promote oral health and prevent diseases thus ensure good oral health; based on the elements of the primary health care approach. It is directed at all citizens of South Africa (SA) including service providers such as oral and general health workers, and the government oral health directorate. The goals also strive to ensure that all people have access to quality oral health care at affordable rates and that oral health services are provided by competent clinicians, using up to date technology in facilities that are safe. ${ }^{8}$

The promotion of oral health entails education and preventive services at the primary health care level and includes schools. These are the services that are rendered at schools, and form part of the Integrated School Health Programmes (ISHPs) through partnerships with academic institutions, Department of Health and private oral health companies.

The prevention of oral disease requires screening for early detection and management. This includes the prevention of progression and management of diagnosed diseases. This requires a well-structured referral system for patients to be treated.

The objectives that were included in the NOHPSA were based on previous studies that were relevant at the time during which the policy was developed. However, with time these targets need to be amended and modified to be relevant and in line with the current disease burdens. In order to determine whether the targets, in any policy are relevant, it is important to carry out surveys carry out surveys that will measure the disease burden and the impact of services rendered. The most recent oral health survey of South Africa was focused on children, and it was conducted between 1999 and 2002. During that period $60.3 \%$ of children under age 6 presented with caries and $80 \%$ of that cohort had untreated caries. ${ }^{13}$
This implies that there is a high prevalence of dental caries among children and that the vast majority of these children do not have access to dental care. Since then, there has been no other National surveys conducted to gauge whether this has changed or not.

Thus the oral health strategy that is currently the operational basis of oral health programmes in the country needs to be reviewed in order to identify weaknesses and improve on them so that when a new policy is developed, it can be more appropriate and relevant to the South African context.

\section{AIM}

The aim of this study was to review the National Oral Health Policy of SA (NOHPSA) in terms of the context, strengths, weaknesses, implementation and monitoring with regards to the oral health status of the South African population.

\section{METHODS}

This review was conducted using aspects of the conceptual framework developed by Singh et al. (2010) which was specifically designed for the analysis of oral health promotion in health policies. ${ }^{3}$

Their frame was adapted from the advocacy coalition frameworks. Using it as a guide one understands that to analyse an oral health policy one must understand the political and health transformations of the country, in so doing also be cognisant of the possibility of unexpected changes to that landscape, while taking into consideration the healthcare belief systems of the stakeholder who are affected.

The objectives of the policy were analysed based on the SMART criteria. ${ }^{4}$ These criteria ensure that the goals are 


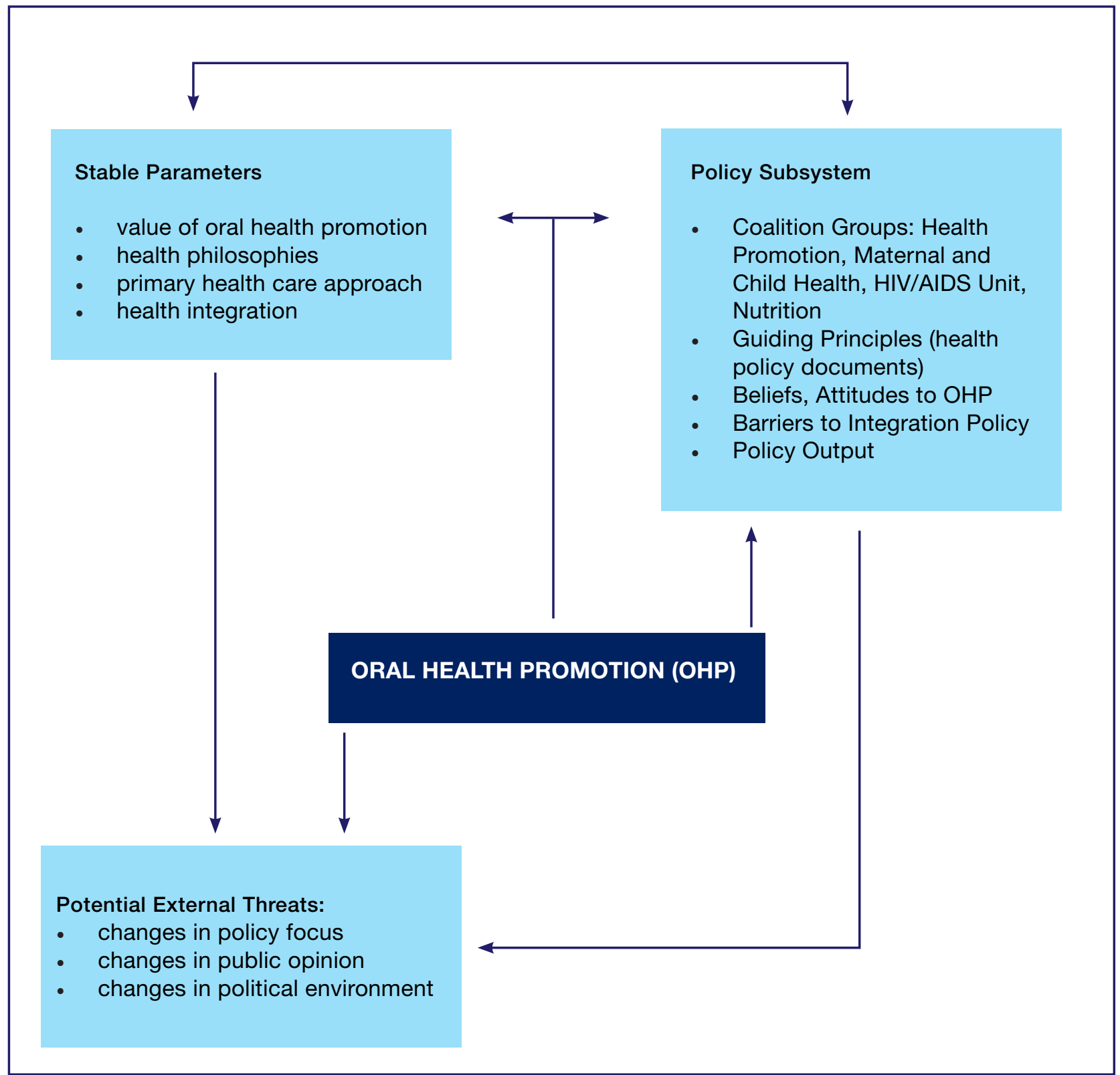

Figure 2. Adapted conceptual frame work ${ }^{3}$

Specific, Measurable, Relevant and Timely. It is a thorough analysis of the policy document pertaining to context, implementation and monitoring.

\section{RESULTS}

The education and preventive services places emphasis on the fluoridation of water for communities as a pivotal preventive programme. However, to date no province or district has introduced a water fluoridation program. Other preventative services that should be implemented are unfortunately not defined, however, there are fissure sealants and brushing programmes being conducted throughout the country under the auspices of the department of oral health. ${ }^{15-16}$

The curative services are defined with a focus on prioritisation of the waiting list system. Levels of treatment are set within the scope of practice of oral health professionals and include education, preventive and curative services (management of pain and sepsis set as priority). There is a defined recommendation of the oral health workforce allocation in the form of ratios of oral health professionals per number of people in the country. Regarding dental specialists, however there is no mention of any dental specialists except that of Community Dentistry. The policy defines the scope of the Community Dentist specialist as being confined to non-clinical work including policy development and screening.

The fee structure for these services in the public sector is clearly defined. It is evident that there is a range of free dental services within the lower levels of care and tariffs will be set for higher levels of care. The levels of care are clearly stipulated, from self-care, primary, secondary and tertiary care. All dental services are free at PHC facilities but there is a user fee at secondary and tertiary hospitals for all dental care. The policy differentiates two main areas in the delivery of services; organised services, which are free to stipulated categories, and non-organised services. The organised services are clearly defined as preventive, 
educative and emergency services for children at centres of learning from pre-school through to high school, at schools and at clinics. These include services at state institutions, hospitals and prisons.

Non-organised services include outpatient services, such as restorations, prosthodontic, orthodontic treatment and other elective treatments that are not inclusive of relief of pain, sepsis and trauma.

The objectives in the policy as a target for the year 2000, have included all age cohorts benchmarked on the South African Statistics from the 1980's in line with global oral health promotion standards. ${ }^{3}$

1. 1-6 year old cohort aimed at $50 \%$ caries free by 2000 .

2. 12 year old cohort must have DMFT of 1.5 or less.

3. 20 year old cohort must have a $60 \%$ tooth retention and have at least 35\% increased periodontal health.

4. 35-44 year old cohort must have a reduction in the level of edentulism by $6.22 \%$ and ensure at least $15 \%$ are healthy.

5. 60-64 year old cohort must reduce edentulism by $21.41 \%$ and reduce deep periodontal lesions.

The implementation plan is not well structured and does not have clear guidelines. There is no monitoring and evaluation plan.

\section{DISCUSSION}

The policy details goals and targets that are aimed at promoting oral health and thus ensuring that the country benefits from services tailored for that purpose.

However, the policy is focused on addressing the oral health problems but does not include the effects of comorbidities (diabetes, HIV/AIDS etc), lifestyle choices (smoking, alcohol drug abuse) and other determinants of health (socioeconomic factors, malnutrition and trauma) that may predispose individuals to oral diseases. ${ }^{17-21}$ Nor does it mention a collaborative prevention and oral health promotion with the medical professionals. The policy mentions collaboration with South African Medical Services only with reference to data collection. Other medical professionals such as paediatricians, speech and hearing therapists, pharmacists, medical doctors, etc. could play a vital role in the prevention and education related to oral diseases..$^{22-24}$ There is no mention of a private public partnership, nor the role of the private sector in provision of oral health care in the country.

\section{Promotive and preventive services}

The education and preventive services are not detailed enough. The water fluoridation rhetoric is redundant as SA has not implemented water fluoridation and some areas that use borehole water have water with fluoride at levels of $25 \mathrm{ppm}^{25}$ which is way over the recommended dose of $1 \mathrm{ppm}$. There are a lot of controversial views pertaining to fluoridation of water and particularly on the African continent ${ }^{26}$. Other feasible means of delivery of fluoride should be explored such as toothpaste. There is also no mention of interdisciplinary collaboration with nurses, family medicine practitioners, dieticians etc, in relation to oral health promotion.
Free services at point of contact especially at primary health care level increase accessibility to services and draws closer to reducing the health inequalities experienced in the country. ${ }^{27-28}$ With a user fee being charged at higher levels of care, an appropriate referral system would ensure that patients do not incur costs for treatment they may have not needed but wanted. In the policy it is stated that to gain access to secondary and tertiary oral health care, one must be referred via the hierarchy. As it is currently, all levels are accessible without referral, which is contrary to what is stated. ${ }^{29}$ Adhering to the policy in this regard would aid in reducing the burden of demand at secondary and tertiary public health facilities, significantly reduce waiting times and may lead to saving on operational costs. ${ }^{29}$ Access to appropriate oral health services should be emphasised on with the consideration of where people live and their socio-economic statuses, such that rural settings are far off the standard and quality of urban settings. Appropriate technology should make it possible to bridge the gap between the two settings, thus achieving equitable access ${ }^{30}$ thus ensuring that every individual receives services that they need when they need them.

\section{Provision of Oral Health Services}

This section deals with the different types of services and categories of oral health providers. The services are divided into two categories, organised and non organised services. Organised services stipulates a clear mandate to cater for young children without description of exact services. The authors recommend that fissure sealants be included in the policy as one of the services rendered to the school learners as per other studies. ${ }^{11,31-32}$ The non-organised services are vaguely described as outpatients with pain, sepsis and trauma. Clarity is needed as to whether this is within general health or dental clinics. It should be specified at which level of care the outpatients' services will be provided. Currently, these services are being provided at dental clinics at primary, secondary and tertiary facilities.

In order to achieve efficient curative services, a sufficient workforce per population is required and currently studies have shown that there is a shortage of human resources in oral health. ${ }^{33}$ The personnel norms as stipulated in the policy definitely need to be revised and reviewed as they are outdated. Both the population and the workforce has increased considerably since the 1990's. ${ }^{33}$ Therefore an audit of the population and human resources need to be done to determine the type and number of human personnel that need to be trained. The $\mathrm{HRH}$ strategy for 2030 indicates there may be surplus of dentists and a shortage of dental therapists whose scope is better aligned for the PHC package. ${ }^{34}$

The policy stipulates that Community Dentistry specialist's scope should be confined to non-clinical duties. It is not clear in the policy why that statement stands as there is no supporting evidence to it, their scope of practice is not stated as such. The assumption is that it may be owing to the fact that as of 2015 there were only 36 Community Dentistry Specialists available for service at the ratio of $1: 1$ 636424,33 therefore at the time the draft was made the numbers were even smaller. Hence the policy makers saw it fit to limit the Community Dentistry scope of practice to non-clinal duties. However, it is essential for Community dentistry specialists to be involved in research, screening 
of patients, treating patients where necessary and providing insight and knowledge on planning future policies. Ideally they should also inform oral health strategies and see to the monitoring and evaluation of implemented programmes. ${ }^{35}$

Mention must also be made of the role of other specialists including dental practitioners, oral hygienists and dental therapists and their numbers required to ensure the oral health targets can be achieved with adequate human resources.

\section{Infrastructure and resources}

Physical facilities, equipment, instruments and consumable supplies are mentioned to be procured through tenders. Emphasis should be placed on assuring that these resources are equitably distributed. The information is not detailed enough for any individual in the oral health care workforce to be able to initiate or follow up on procuring of items for clinics. The NOHPSA makes mention of accommodation needs and planning for oral health services in accordance with other policies, yet there is no detail as to which policies these are.

\section{Objectives}

Objective 1 was for $50 \%$ of the 6 year age group to be caries free by 2000 , it has not yet been achieved. As of the most recent National Oral Health survey, only $30.7 \%$ of this population remained caries free by the year $2000 .{ }^{13}$ The socio-economic status of the country renders many citizens categorised as underprivileged and under serviced. The quality of the diet is directly associated with socio-economic status. ${ }^{5}$ Lack of oral health services and resources, lack of evidence of implementation of strategies for oral health education programmes and a multi-sectoral approach to prevention amplifies the problem of children not receiving quality oral health services. ${ }^{6-7,9-12,36}$ Poor diet is linked to poor oral health, if this narrative remains the same it may be near impossible to reach the target aimed at reduction of caries in children. Even in light of the implementation of the National Health Insurance $(\mathrm{NHI})$ Bill which aims to increase access to oral health services for all.

Objective 2 was to obtain a DMFT of 1.5 or less for the 12 year old group and this was achieved by $2004^{13}$. At the time that the objective was set, the figures were benchmarked against the National Oral Health survey of 1982 when the DMFT of the cohort was recorded at 2.5, by the next oral health survey that was published in 2004 it was at 1.1. This may be attributed to the improvement of school oral health programmes over the years. ${ }^{37-38}$ There are no new surveys to confirm the current national prevalence.

Objective 3 was to ensure that at least $60 \%$ of 20 year olds retain all their teeth and at least 35\% of them have a minimum of three healthy sextants. Surveys need to be conducted to measure the current oral health status among the 20 year olds.

Objective 4 mentions reduction of edentulism from 10.36\% to $6.2 \%$, to ensure that $80 \%$ retain at least 20 of their teeth and with respect to periodontal status, $15 \%$ have 3 healthy sextants. These targets are set in percentages but with no reference to the population size. It is difficult to assess whether this objective was achieved or not. The objective needs to be reviewed based on evidence and redefined in line with recent survey data.
Objective 5 is to reduce edentulism from $26.76 \%$ to $21.41 \%$, ensure that $60 \%$ retain 20 teeth and reduce the mean number of deep pockets to 0.27 . Similar to the fourth objective, these percentages were set without a population size reference. It must be taken into consideration that this age group often requires removable dental prostheses. This being attributed to the fact that the current services for adults are predominantly extractions due to a possible lack of infrastructure, materials and equipment. ${ }^{11}$ Currently the state covers the costs of these prostheses for pensioners in order to restore function. Yet, there are few dentures being delivered in PHC clinic and in rural areas. However, the ideal would be for this status quo to be changed by ensuring that periodontal knowledge is emphasised in the oral health education programmes, and periodontal treatment initiated from the onset rather than at a later stage.

Objective 6 and 7 address the need to obtain and collate data on oral pathology and trauma related conditions. Various academic institutions are well underway with reporting and dissemination of publications pertaining to that. It would be beneficial for routine continuous professional development sessions to be conducted with private dentists and public dentists in this regard, for them to be encouraged to report their findings.

Re-engineering of primary health care services as set out in the $\mathrm{NHI}$ can ensure that each indicator is achieved by ensuring an equitable distribution of manpower to service each oral health facility and the funding to maintain the equipment. This would see to the reduction of the prevalence of edentulism by encouraging the provision of restorative procedures over extractions and changing the mindset of the population with regards to extractions versus restorations. ${ }^{39-40}$

\section{Implementation}

The implementation plan is vaguely structured, without a budget nor direct guidelines as to how to be implemented. It simply states the levels at which the policy should be implemented but not so much by which portfolios. All service providers of oral health are expected to implement this NOHPSA when rendering clinical services. All training and research facilities including the medical services and the private sector should develop and implement their own internal policies based on the NOHPSA.The NOHPSA should further aid in advocating for creating environments that are conducive to adoption of this policy, with training for appropriate management to oversee it. The fiscal aspects of the policy should be elaborated under implementation, as there will be a need to ensure that resources are allocated appropriately to achieve the objectives set in the main policy. The general public is privy to the contents of the policy, this will ensure improved utilisation and avoid misuse of oral health facilities. This will also ensure that oral health professionals do not abuse their authority when providing oral health care.

There is no mention on strategies to monitor and evaluate the policy. A review of the policy conducted in 2010, recognises the emphasis on equity, health promotion, integration and focus on the primary health care approach. ${ }^{3}$ There is however little documented evidence on implementation of this policy, hence there is a need for the policy to be re-evaluated. 


\section{CONCLUSION}

The policy context is in line with global oral health policies in that the aim is to provide universal oral health coverage for all with the end goal of enabling populations to take ownership of own oral health care.

The policy strengths lie on the following intentions:

- Training human resources to cope with demand

- Involve the community in planning

- Implementation based on evidence and thus tailor make programs to need

- Make oral health services accessible to all

- Make oral health services affordable to all

- Enable communities to practice self-care

However, there is no evidence of any of the intentions being translated into action. That poses as a significant weakness, along with the fact that oral health promotion is dominated by oral health professionals in a system that has a curative dominance over preventive care. Intersectoral collaboration with medical health disciplines to address oral health conditions could assist in changing that narrative.

The fact that there is no mention of monitoring and evaluation, implies that there is little effort to collate periodic data that each facility in the country is mandated to submit. The failure to collate data may also be attributed to lack of uniformity in terms of dental records within the country. It is thus impossible to review the implementation of the policy.

Overall, the policy was clear as to its outcomes but did not provide clear guidelines as to achieving these outcomes. This is inappropriate in a country like SA, where there is widespread disparity between provinces and even within provinces. The disease burden and demand differ across the country and as such a preventive measure which is effective in one community might not be in another. Therefore, the policy is not directive in nature and allows provinces and districts the leeway to adopt measures that will be suitable for their respective communities. However, the government of South Africa must be commended in drawing up such a policy which gives direction and a road map to where oral health should go. Many other countries do not have such a policy and hence the government has achieved a policy that is practical and implementable.

\section{Recommendations}

The policy framework is sound but needs critical emphasis on four areas, research, budget, multisectoral collaboration and a monitoring and evaluation plan.

It is not possible to set goals without relevant and up to date data. This hinges on oral health surveys that are conducted at local levels then collated in order to establish the prevalence of oral conditions and distribution of oral health personnel and facilities throughout the country.

Continuous professional development courses for all health care workers will ensure that they are well equipped with updated knowledge. Ensuring that if new technology is introduced for service rendering, staff members are able to incorporate it into the services, such that it does not become a futile expense. The facilities themselves should be well maintained to ensure that they are safe for the use of patients and staff.

An annual budget must be set for the intended goals, this would require for it to be defined at National and provincial levels. The allocation of funds should be done equitably amongst districts and provinces.

Take on a multisectoral approach to incorporate aspects of policies that influence oral health. Using the common risk factor approach to incorporate oral health into health policies. This can be achieved by providing lectures to allied health professionals during CPD meetings to educate them on oral health.

Establish a transparent monitoring and evaluation process for setting goals. This will allow for officials to take ownership of delegated responsibilities and also be accountable for its successes and failures. Not only will this ensure that policy objectives are are met but it may also act as a motivational tool for oral health teams involved.

\section{References}

1. Drachev SN, Brenn T, Trovik TA. Dental caries experience and determinants in young adults of the northern state medical university, arkhangelsk, north-west russia: A cross-sectional study. BMC Oral Health. 2017; 17(1):136.

2. Fedorenko $V$. The idea of maintaining and improving the health of our nation is professor iryna datsenko's life motto. Proceeding of the Shevchenko Scientific Society. Medical Sciences. 2019; 55(1):164-72.

3. Singh S, Myburgh NG, Lalloo R. Policy analysis of oral health promotion in south africa. Global health promotion. 2010; 17(1):16-24.

4. Les MacLeod EdD M. Making smart goals smarter. Physician executive. 2012; 38(2):68.

5. Similä M. The norbagreen 2002 study: Consumption of vegetables, potatoes, bread and fish in the nordic and baltic countries: Nordic Council of Ministers; 2003.

6. Reddy $M$, Singh S. The promotion of oral health in health-promoting schools in kwazulu-natal province, south africa. South African Journal of Child Health. 2017; 11(1):16-20.

7. Mothupi KA, Nqcobo CB, Yengopal V. Prevalence of early childhood caries among preschool children in johannesburg, south africa. Journal of Dentistry for Children. 2016; 83(2):83-7.

8. Department of Health, Western Cape. South African National Oral Health Strategy. https://www.westerncape. gov.za/text/2003/national_policy_oral_health_sa.pdf (Accessed April 2021)

9. Mthethwa J, Mahomed O. Predictors of dental caries among adults attending public oral health facilities in two districts in kwazulu-natal, south africa: A cross sectional study. 2020;

10. Nacobo C, Ralephenya T, Kolisa YM, Esan T, Yengopal V. Caregivers' perceptions of the oral-health-related quality of life of children with special needs in johannesburg, south africa. Health SA Gesondheid (Online). 2019; 24:1-7.

11. Bhayat A, Madiba TK, Nkambule NR. A three-year audit of dental services at primary health care facilities in gauteng, south africa: 2017 to 2019. Journal of International Society of Preventive \& Community Dentistry. 2020; 10(4):452. 
12. Motloba P, Makwakwa N, Machete L. Justice and oral health-implications for reform: Part two. South African Dental Journal. 2019; 74(4):208-11.

13. van Wyk PJ, van Wyk C. Oral health in south africa. International Dental Journal. 2004; 54(S6):373-7.

14. Department of Health. South African National Oral Health Strategy. Pretoria 2004 .p.1-31

15. National Department of Health and Department of Basic Education in South Africa. Intergrated school health policy. 2012. p. https://serve.mg.co.za/content/ documents/2017/06/14/integratedschoolhealthpolicydbeanddoh.pdf Accessed online 16 August 21.

16. Molete M, Stewart A, Igumbor J. Implementation fidelity of school oral health programs at a district in south africa. PLoS One. 2020; 15(11):e0241988.

17. Abanto J, Carvalho TS, Mendes FM, Wanderley MT, Bönecker M, Raggio DP. Impact of oral diseases and disorders on oral health冈related quality of life of preschool children. Community dentistry and oral epidemiology. 2011; 39(2):105-14.

18. Cagetti MG, Wolf TG, Tennert C, Camoni N, Lingström $\mathrm{P}$, Campus $\mathrm{G}$. The role of vitamins in oral health. A systematic review and meta-analysis. International Journal of Environmental Research and Public Health. 2020; 17(3):938.

19. Greenspan JS, Challacombe SJ. The impact of the world workshops on oral health and disease in hiv and aids (1988-2020). Oral Diseases. 2020; 26:3-8.

20. Rahman N, Walls A. Nutrient deficiencies and oral health. The impact of nutrition and diet on oral health: Karger Publishers; 2020. p. 114-24.

21. Birch S, Listl S. The economics of oral health and health care. Max Planck Institute for Social Law and Social Policy Discussion Paper. 2015; (07-2015)

22. Park SE, Donoff RB, Saldana F. The impact of integrating oral health education into a medical curriculum. Med Princ Pract. 2017; 26(1):61-5.

23. Atchison KA, Weintraub JA, Rozier RG. Bridging the dental-medical divide: Case studies integrating oral health care and primary health care. The Journal of the American Dental Association. 2018; 149(10):850-8.

24. Fiorillo L. Oral health: The first step to well-being. Multidisciplinary Digital Publishing Institute; 2019.

25. Thakuria D, Godboley BJ. Contamination and removal of iron and flouride from groundwater by adsorption and filtration: A review. IJSTE; 2016.

26. Steier L, Steier R, Steier G. Intersections of dental health and food law: The conflict of systemic flouridation as a public health instrument to prevent tooth decay. International food law and policy: Springer; 2016. p. 291-318.

27. South African National Department of Health. Human Resources for Health South Africa: HRH Strategy for the Health Sector: 2012/13-2016/17. Pretoria: NDoH; 2011.

28. Burger R, Christian C. Access to health care in post-apartheid south africa: Availability, affordability, acceptability. Health Economics, Policy and Law. 2020; 15(1):43-55.

29. Goudge J, Gilson L, Russell S, Gumede T, Mills A. The household costs of health care in rural south africa with free public primary care and hospital exemptions for the poor. Tropical medicine \& international health. 2009; 14(4):458-67.

30. Ayo-Yusuf O, Ayo-Yusuf IJ. The role of socio-economic position on satisfaction with oral health services among south african adults: A structural equation model. South African Dental Journal. 2016; 71(10):442-6.

31. Molete M, Chola L, Hofman K. Costs of a schoolbased dental mobile service in south africa. BMC health services research. 2016; 16(1):590.

32. Molete M, Stewart A, Bosire E, Igumbor J. The policy implementation gap of school oral health programmes in tshwane, south africa: A qualitative case study. BMC health services research. 2020; 20:1-11.

33. Bhayat $A$, Chikte $U$. Human resources for oral health care in south africa: A 2018 update. International journal of environmental research and public health. 2019; 16(10):1668.

34. National Department of Health. 2030 human resources for health strategy: Investing in the health workforce for universal health coverage. March 2020.

35. Ramphoma K. Oral health in south africa: Exploring the role of dental public health specialists. South African Dental Journal. 2016; 71(9):402-3.

36. Shaikh RB. Knowledge, attitude and practices towards preventive dentistry amongst dental clinicians in gauteng department of health. 2019;

37. Shung-King $M$, Orgill M, Slemming W. School health in south africa: Reflections on the past and prospects for the future. South African health review. 2013; 2013(1):59-71.

38. Shasha $Y$, Taylor M, Dlamini S, Aldous-Mycock C. A situational analysis for the implementation of the national school health policy in kwazulu-natal. Development Southern Africa. 2011; 28(2):293-303.

39. Smit D, Barrie R, Louw A. The burden of dental caries in the western cape and a recommended turn-around strategy. South African Dental Journal. 2017; 72(8):360-5.

40. Smit D, Osman Y. The availability of the basic oral health care package in the western cape. South African Dental Journal. 2017; 72(6):258-61. 